\title{
Manifestations buccales de la sclérose tubéreuse de Bourneville
}

\author{
J. Lafont (Marseille), J.H. Catherine (Marseille), M. Lejeune (Marseille), U. Ordioni \\ (Marseille), R. Lan (Marseille), F. Campana (Marseille)
}

L'objectif de ce travail est de faire le point sur les manifestations buccales de la sclérose tubéreuse de Bourneville (STB) à travers le cas d'un jeune patient. Un jeune homme de 15 ans était adressée pour la mise en place de minivis orthodontique afin de fermer des espaces d'agénésies de 35 et 45. L'interrogatoire retrouvait une STB dont les manifestations épileptiques étaient traitées par de la lamotrigine $75 \mathrm{mg} / \mathrm{j}$ et de la carbamazépine LP $200 \mathrm{mg} / \mathrm{j}$. L'examen clinique exo-buccal retrouvait des macules hypochromiques sur le membre inférieur droit, des angiofibromes faciaux et une malformation vasculaire jugale gauche. L'examen endo-buccal retrouvait de multiples lésions buccales sur les papilles interdentaires pouvant évoquer des fibromes ou des hamartomes. Une biopsie était réalisée et retrouvait un revêtement malpighien, discrètement hyperplasique et sans atypie cellulaire. Les faisceaux collagènes du conjonctif étaient mêlés à de nombreux fibroblastes aux noyaux réguliers, sans mitose visible.

Les cellules inflammatoires, essentiellement mononuclées, étaient dispersées mais tendaient à se regrouper autour de vaisseaux nombreux et hyperplasiques. L'examen concluait à un fibrome. Aucun traitement buccal n'était proposé devant l'absence de symptôme et de demande esthétique. La STB est une maladie génétique autosomique dominante avec une incidence de 1/10 000. Elle est liée à une mutation du gène TSC1 sur le chromosome 9 ou du gène TSC2 sur le chromosome 16 qui perturbe la sécrétion d'une protéine régulant la voie mTOR. C'est une maladie multisystème avec une expression clinique variable. Les principaux symptômes sont l'épilepsie, le retard mental et la présence d'adénomes sébacés, mais la maladie est associée à un polymorphisme clinique rendant le diagnostic difficile. La conférence de consensus de 2012 a ainsi défini des critères diagnostiques majeurs (lésions cutanées, oculaires, cérébrales, cardiaques, pulmonaires, rénales,..) et mineurs dont deux sont bucco-dentaires. Le diagnostic est retenu devant deux critères majeurs ou un critères majeur et deux critères mineurs. Les signes oraux sont la présence de trois ou plus puits d'émail et deux ou plus fibromes gingivaux. Les fibromes gingivaux atteindraient 50 à $70 \%$ des patients. La région antérieure maxillaire semble la plus touchée. L'exérèse est indiquée en cas de gêne esthétique ou de saignements associés. Actuellement, les inhibiteurs de mTOR représentent une option thérapeutique proposée dans la prise en charge des patients atteints de STB. La STB est une pathologie rare. La présence de lésions buccales fait partie des critères diagnostiques. Bibliographie

1- Northrup H, Krueger DA. Tuberous sclerosis complex diagnostic criteria update: recommandations of the 2012 international tuberous sclerosis complex consensus conference. Journal of pediatricneurology. $2013 ; 49(4): 243-254$.

2- Nico MM, Hammerschmidt M, Lourenço SV. Oral mucosal manifestations in some genodermatoses: correlation with cutaneous lesions. Eur J Dermatol. 2013 Sep-Oct;23(5):581-91.

3- Harutunian K, Figueiredo R, Gay-Escoda C. Tuberous sclerosis complex with oral manifestations: a case report and literature review. Med Oral Patol Oral Cir Bucal. 2011 Jul 1;16(4):e478-81 